\title{
Transient Suppression of Ipsilateral Primary Somatosensory Cortex during Tactile Finger Stimulation
}

\author{
Yevhen Hlushchuk ${ }^{1,2}$ and Riitta Hari ${ }^{1,2,3}$ \\ ${ }^{1}$ Brain Research Unit, Low Temperature Laboratory, and 2Advanced Magnetic Imaging Centre, Helsinki University of Technology, 02150 TKK, Espoo, \\ Finland, and ${ }^{3}$ Neuroscience Center, University of Helsinki, 00014 Helsinki, Finland
}

The whole human primary somatosensory (SI) cortex is activated by contralateral tactile stimuli, whereas its subarea 2 displays neuronal responses also to ipsilateral stimuli. Here we report on a transient deactivation of area $3 \mathrm{~b}$ of the ipsilateral SI during long-lasting tactile stimulation.

We collected functional magnetic resonance imaging data with a $3 \mathrm{~T}$ scanner from 10 healthy adult subjects while tactile pulses were delivered at 1,4 , or $10 \mathrm{~Hz}$ in $25 \mathrm{~s}$ blocks to three right-hand fingers. In the contralateral SI cortex, activation [positive blood oxygenation level-dependent (BOLD) response] outlasted the stimulus blocks by $20 \mathrm{~s}$, with an average duration of $45 \mathrm{~s}$. In contrast, a transient deactivation (negative BOLD response) occurred in the ipsilateral rolandic cortex with an average duration of $18 \mathrm{~s}$. Additional recordings on 10 subjects confirmed that the deactivation was not limited to the right SI but occurred in the SI cortex ipsilateral to the stimulated hand. Moreover, the primary motor cortex (MI) contained voxels that were phasically deactivated in response to both ipsilateral and contralateral touch.

These data indicate that unilateral touch of fingers is associated, in addition to the well known activation of the contralateral SI cortex, with deactivation of the ipsilateral SI cortex and of the MI cortex of both hemispheres. The ipsilateral SI deactivation could result from transcallosal inhibition, whereas intracortical SI-MI connections could be responsible for the MI deactivation. The shorter time course of deactivation than activation would agree with stronger decay of inhibitory than EPSP at the applied stimulus repetition rates.

Key words: fMRI; BOLD; somatosensory cortex; motor cortex; tactile; human

\section{Introduction}

In intracranial recordings, area $3 \mathrm{~b}$ of the human primary somatosensory (SI) cortex is activated only by contralateral tactile stimuli, whereas the more posterior parietal cortex (possibly areas 1, 2, and 5) may display short- and long-latency responses also to ipsilateral activation of the somatosensory afferents (Allison et al., 1989). In functional magnetic resonance imaging (fMRI) studies, median nerve stimulation activates, besides the contralateral SI, also the ipsilateral SI area 2 and/or the posterior parietal area 5 (Nihashi et al., 2005).

In magnetoencephalographic recordings, long-latency (90$300 \mathrm{~ms}$ ) responses have been reported in the ipsilateral SI region to electric median nerve stimuli in healthy adults (Korvenoja et al., 1995, 1999). However, such findings are rare: only 13 of 401 neurological patients and only 1 of 81 healthy subjects showed ipsilateral responses to median nerve stimulation at area $3 \mathrm{~b}$ of SI (Kanno et al., 2003). All ipsilateral SI responses do not reflect ipsilateral projections; for example, a prominent ipsilateral $58 \mathrm{~ms}$

\footnotetext{
Received Dec. 24, 2005; revised March 31, 2006; accepted April 18, 2006.

This study was supported by the Academy of Finland, the Ministry of Education Finland via the Finnish Graduate School of Neuroscience, and the Sigrid Jusélius Foundation. We thank Dr. Cristina Simões-Franklin and Nuutt Vartiainen for active participation in the fMRI measurements and Dr. Jesper Andersson and Dr. Cyril Pernet for valuable discussions. We are also grateful to Dr. Christoph Braun for kindly providing us the original psychophysical data of Braun et al. (2005).

Correspondence should be addressed to Dr. Yevhen Hlushchuk, Brain Research Unit, Low Temperature Laboratory, Helsinki University of Technology, P.0. Box 2200, FIN 02015 HUT, Espo0, Finland. E-mail:yevhen@neuro.hut.fi. DOI:10.1523/JNEUROSCI.5536-05.2006

Copyright $\odot 2006$ Society for Neuroscience $\quad$ 0270-6474/06/265819-06\$15.00/0
}

response followed unilateral finger tapping, reflecting tactile contamination to the other hand (Hari and Imada, 1999).

Activity changes in ipsilateral hemisphere have also been reported during unilateral finger movements or low-force grip tasks in fMRI recordings (Allison et al., 2000; Nirkko et al., 2001; Hamzei et al., 2002; Stefanovic et al., 2004; Newton et al., 2005). Although these studies typically emphasized deactivation of the ipsilateral primary motor cortex (MI) (Hamzei et al., 2002; Stefanovic et al., 2004; Newton et al., 2005), detailed examination of the published data suggests that the deactivation clusters included the ipsilateral SI cortex.

Such confounding between SI and MI cortices could result from proprioceptive and tactile feedback associated with hand movements. Moreover, because MI and SI are located on the opposite banks of the same (central) sulcus, the typical spatial smoothing applied in fMRI studies can blur the activations beyond the border. The difficulty of anatomical separation of SI and MI activations could be overcome by additional functional distinction. We therefore aimed to study fMRI changes in the ipsilateral SI cortex by applying pure tactile stimuli to fingers of subjects who kept their hands immobile.

Parts of this work have been published previously in abstract form (Hlushchuk and Hari, 2005).

\section{Materials and Methods}

This study comprised experiment 1 as well as experiment 2, designed on the basis of the results of experiment 1.

Subjects, stimuli, and experimental conditions. fMRI signals were re- 
corded from healthy adults: 10 subjects in experiment 1 (seven males, three females; mean age, 27 years; range, 23-33 years) and 10 subjects in experiment 2 (five males, five females; mean age, 29 years; range, $24-45$ years; four of these subjects had also participated in experiment 1). The experimental protocol had received previous approval by the local ethics committee, and informed consent was obtained from all subjects before the experiment.

Tactile stimuli were delivered to fingers by balloon diaphragms driven by compressed air (Mertens and Lütkenhöner, 2000). Each stimulus lasted for $282 \mathrm{~ms}$ ( $40 \mathrm{~ms}$ rise time, $62 \mathrm{~ms}$ plateau, $180 \mathrm{~ms}$ return to baseline pressure). In experiment 1 , the stimuli were delivered in a random order to the index, middle, and ring fingers of the right hand, at 1,4 , or $10 \mathrm{~Hz}$ in $25 \mathrm{~s}$ blocks; the blocks were separated by rest periods of same duration. The blocks with different stimulus frequencies were presented in a random order within the sequence, which altogether comprised 15 stimulation blocks (five per frequency) alternating with 16 rest periods. The total duration of the sequence was $13 \mathrm{~min}$.

In experiment 2, tactile stimuli were delivered unilaterally to either the left or right hand in separate blocks at frequencies from 1.7 to $5 \mathrm{~Hz}$. The pseudorandomly arranged unilateral $20 \mathrm{~s}$ stimulation blocks alternated with $20 \mathrm{~s}$ rest blocks, and the total 7 min sequence comprised five stimulation blocks per hand and 11 rest blocks.

The presentation and timing of the stimuli was controlled by Presentation software (version 0.60) running on a personal computer.

MRI data acquisition and analysis. Functional MRI was performed on a Signa 3T MR scanner (GE Medical Systems, Milwaukee, WI) using gradient-echo (GRE) planar imaging (EPI) sequence. In experiment 1 , a standard head coil was used with the following parameters of the GREEPI sequence: flip angle (FA), $90^{\circ}$; time of repetition (TR), $2500 \mathrm{~ms}$; time to echo (TE), $32 \mathrm{~ms}$; field of view (FOV), $200 \mathrm{~mm}$; matrix, $64 \times 64$; number of excitations (NEX), 1 . The whole brain was covered with 31 contiguous axial-oblique slices that were collected in an interleaved manner (slice thickness, $4 \mathrm{~mm}$ ). In experiment 2 , the same MRI system was used after EXCITE upgrade with an eight-channel head coil. The imaging parameters for the GRE-EPI sequence were as follows: FA, 85; TR, 2000 $\mathrm{ms}$; TE, $32 \mathrm{~ms}$; FOV, $200 \mathrm{~mm}$; matrix, $64 \times 64$; NEX, 1 . The whole brain was covered with axial-oblique slices, as in experiment 1.

From the 314 volumes in experiment 1 and from the 214 volumes in experiment 2 , the first four were omitted from the statistical analysis to include only volumes with full magnetic saturation.

For all subjects, anatomical three-dimensional (3-D) images were obtained in the sagittal plane with 3-D fast spoiled gradient echo sequence (inversion recovery prepared): TR, $9 \mathrm{~ms}$; TE, $1.9 \mathrm{~ms}$; FA, $15^{\circ}$; $256 \times 256$ matrix; slice thickness, 1.3 or $1.4 \mathrm{~mm}$ in experiment 1 and $1.0 \mathrm{~mm}$ in experiment 2 .

The acquired fMRI data were analyzed with BrainVoyager QX (BV QX) software (Brain Innovation, Maastricht, The Netherlands). Preprocessing included 3-D motion correction, high-pass filtering and linear trend removal, and slice scan-time correction. Gaussian spatial smoothing (full-width half-maximum, $6 \mathrm{~mm}$ ) was used in experiment 1 , whereas no spatial smoothing was applied in experiment 2 to avoid any smearing affecting distinction between activations in the anterior and posterior banks of the central sulcus that is between the MI and SI cortices. All fMRI data were normalized to Talairach space (Talairach and Tournaux, 1988), in which all of the additional statistical analyses were performed.

The data were analyzed using general linear model. In experiment 1 , the model was obtained by overlaying the hemodynamic response function (Boynton et al., 1996) onto the boxcar functions of the time courses of the tactile stimulations. The conclusions were based on randomeffects analysis of the whole subject group. False discovery rate (Genovese et al., 2002) at the level of $<0.05$ was used to threshold group statistical maps.

In experiment 2, activity in the SI-MI cortex was further explored by applying a mask that was based on the right SI-MI deactivation cluster observed in experiment 1 . The cluster edges were extended stepwise in all directions by 11 voxels, each $1 \times 1 \times 1 \mathrm{~mm}^{3}$ in size, and the mask for the left hemisphere was created by mirroring the right-hemisphere mask. The mask covered both the anterior and the posterior banks of the central sulcus in both hemispheres and in each subject.

The blood oxygenation level-dependent (BOLD) responses observed in experiment 1 had different time courses: the positive BOLD response outlasted the whole stimulation block, whereas the negative BOLD response was phasic and returned to the baseline level already during the stimulation block. Therefore, for optimal detection of the BOLD responses, different hemodynamic functions were used to model these two responses in experiment 2 (see Fig. 2C). The default hemodynamic response function in BV QX (Boynton et al., 1996) was used to model tonic, long-lasting responses, whereas a custom-built model, based on the mean time course of the deactivation cluster in the ipsilateral rolandic cortex (see Fig. 2 B), was used to model phasic responses.

To verify involvement of the SI cortex in the observed deactivation, conjunction analysis of individual data (at first-level statistics) was applied to detect regions in SI exhibiting tonic activation in response to contralateral tactile stimulation and phasic deactivation in response to ipsilateral stimulation. Because of a strong hypothesis based on experiment 1 , threshold of $p_{\text {uncorrected }}<0.1$ was applied for the conjunction of the contrasts in experiment 2 . In BV QX, this means that detected voxels need to have $p<0.1$ in each of the two contrasts (for the conjunction analysis implemented in BV QX software, see Nichols et al., 2005). Furthermore, additional anatomical confines were applied within the masked region: voxels were taken for SI (MI) activation only if they were active in an appropriate contrast and were located in the posterior (anterior) bank of the central sulcus.

Individual-subject analysis was also applied in experiment 2 to detect regions in the MI cortex (exhibiting phasic deactivation to unilateral stimulation of either the left or right hand). In this analysis, no conjunction was used and the maps were thresholded at $p_{\text {uncorrected }}<0.01$.

When in experiment 2 more than one cluster satisfied all of the criteria in a contrast, a cluster larger in size and/or closer to the hand knob area was preferred and taken into additional analysis.

\section{Results}

\section{Areas responsive to tactile stimulation: experiment 1}

Figure 1 (left) shows group-level activations during tactile stimulation when all stimulation frequencies were pooled and contrasted with baseline. Prominent activations (positive BOLD re- 
Table 1. Details of detected clusters in experiment 1

\begin{tabular}{|c|c|c|c|c|c|c|}
\hline & \multicolumn{3}{|c|}{ Talairach coordinates } & \multirow[b]{2}{*}{ Volume } & \multirow[b]{2}{*}{ BOLD } & \multirow[b]{2}{*}{ Max $t$ value } \\
\hline & $x$ & $y$ & $z$ & & & \\
\hline \multicolumn{7}{|l|}{ Right hemisphere } \\
\hline $\mathrm{SI}$ & $50 \pm 1.9$ & $-23 \pm 1.4$ & $44 \pm 1.3$ & 74 & Positive & 8.68 \\
\hline SII & $49 \pm 5.5$ & $-28 \pm 6.4$ & $21 \pm 2.6$ & 1571 & Positive & 10.92 \\
\hline Rolandic cortex & $36 \pm 3.6$ & $-23 \pm 3.4$ & $54 \pm 3$ & 835 & Negative & -8.57 \\
\hline PPC & $18 \pm 2.7$ & $-62 \pm 1.6$ & $51 \pm 3.2$ & 342 & Negative & -9.99 \\
\hline PPC & $24 \pm 1.3$ & $-53 \pm 1.4$ & $57 \pm 1.3$ & 66 & Negative & -6.83 \\
\hline \multicolumn{7}{|l|}{ Left hemisphere } \\
\hline $\mathrm{SI}$ & $-49 \pm 6.5$ & $-25 \pm 4.7$ & $50 \pm 7.3$ & 6237 & Positive & 17.47 \\
\hline SII & $-43 \pm 6.4$ & $-22 \pm 8.3$ & $18 \pm 4.9$ & 6214 & Positive & 15.14 \\
\hline Precentral region & $-61 \pm 1.9$ & $3.8 \pm 2$ & $28 \pm 2.1$ & 67 & Positive & 6.72 \\
\hline Cerebellum medial & $-9.4 \pm 3.2$ & $-59 \pm 5.3$ & $-14 \pm 1.9$ & 981 & Negative & -7.87 \\
\hline Cerebellum lateral & $-30 \pm 2.2$ & $-43 \pm 1.7$ & $-24 \pm 1.7$ & 155 & Negative & -6.74 \\
\hline Parieto-occipital region & $-22 \pm 2.2$ & $-75 \pm 2.6$ & $28 \pm 2$ & 250 & Negative & -6.68 \\
\hline
\end{tabular}

Mean \pm SD $x, y$, and $z$ coordinates of voxels in the detected clusters in Talairach space. For clusters in experiment 1 (responses to unilateral tactile stimulation), the sign of the BOLD response, positive or negative, is indicated. Volume represents the cluster size in cubic millimeters. PPC, Posterior parietal cortex.
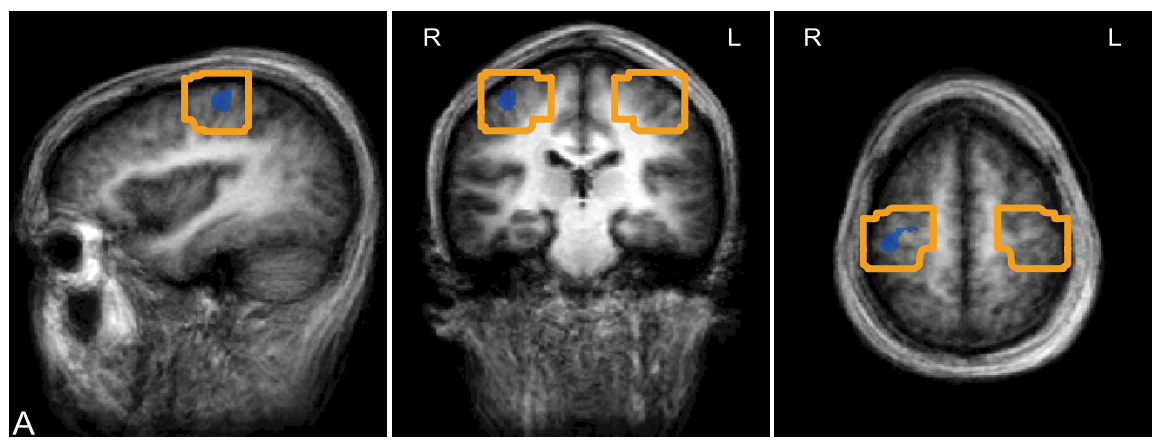

Ipsilateral SI-MI (Expt. 1)

Models of BOLD responses (Expt. 2)
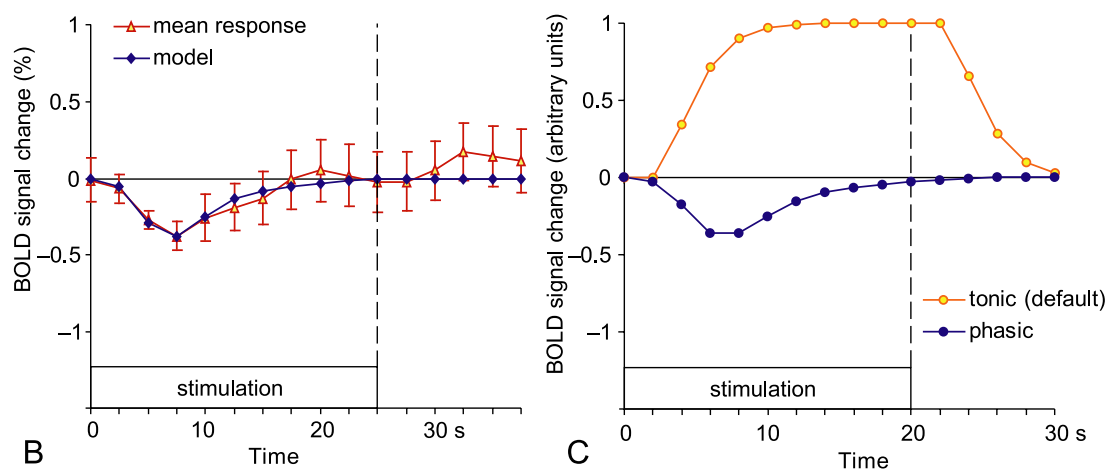

Figure 2. Optimization of the analysis in experiment 2 . $\boldsymbol{A}$, The boxes indicate the volumes of interest, $\sim 30 \mathrm{~cm}^{3}$ around the deactivation cluster observed in the right (ipsilateral) rolandic cortex in experiment 1 and its homolog area in the left hemisphere. $\mathrm{L}$, Left; $\mathrm{R}$, right. $\boldsymbol{B}$, The mean \pm SEM time course (across 10 subjects) of the deactivation cluster (red symbols) and the phasic response model fitted to the data (blue line). $C$, Time courses of the two models: the tonic response model (orange; default in BV QX) and the custom-built phasic response model (blue). Note that the curves are sampled according to the TR used: at $2.5 \mathrm{~s}$ intervals on the left (TR, $2.5 \mathrm{~s}$ in experiment 1) and at 2 s intervals on the right (TR, $2 \mathrm{~s}$ in experiment 2 ).

sponses) are seen in the contralateral (left) SI cortex and in the parietal operculum (SII region) of both hemispheres. An additional small activation cluster is seen in the ipsilateral (right) SI, in the posterior wall of the postcentral gyrus, most likely in cytoarchitectonic area 2 (Grefkes et al., 2001). Moreover, statistically significant deactivation (negative BOLD response) appears in the ipsilateral (right) primary sensorimotor cortex. Smaller deactivation areas were evident in the left parieto-occipital and right posterior parietal cortices, as well as in the contralateral cerebellum (not seen in the figure). Table 1 lists detailed information on the sizes, Talairach coordinates, and maximum $t$ values of the detected activation and deactivation clusters.
The time courses in Figure 1 (right) show the mean \pm SEM (across 10 subjects) BOLD signal changes in both SI cortices at all three stimulation frequencies. In the contralateral SI region, the activation (positive BOLD response) to all stimulation frequencies lasts on average $45 \mathrm{~s}$, which is clearly longer than the 25 s stimulation block. In contrast, a phasic deactivation (negative BOLD response) occurs in the ipsilateral SI cortex with an average duration of $18 \mathrm{~s}$. The positive BOLD responses in the other regions lasted from 42 to $45 \mathrm{~s}$, and the negative BOLD responses lasted from 18 to $25 \mathrm{~s}$.

The mean \pm SEM peak strength of the positive BOLD response in the contralateral SI cortex was $0.77 \pm 0.19 \%$ (averaged across all three frequencies; $p<0.001$ for deviation from 0 level). The peak of the ipsilateral negative BOLD response was $-0.38 \pm 0.17 \%(p<0.05)$. Neither the positive nor the negative BOLD responses showed any clear distinction between the frequencies.

\section{SI areas exhibiting negative BOLD response to ipsilateral and positive BOLD response to contralateral stimulation: conjunction analysis, experiment 2}

Experiment 2 aimed to confirm that the observed deactivation is not hemisphere specific but rather always occurs in the ipsilateral SI cortex. Therefore, the data obtained by stimulation of each hand in different blocks were subjected to conjunction analysis. The conjunctions were designed to reveal areas exhibiting sustained (canonical) positive BOLD responses to contralateral stimulation and phasic negative BOLD responses to ipsilateral stimulation. Figure $2 C$ shows both of these hemodynamic response functions; the custom-built model was based on the average time course of the deactivation cluster in ipsilateral rolandic cortex in experiment 1 (Fig. $2 B$ ).

The analysis was restricted to the volume of $\sim 30 \mathrm{~cm}^{3}$ around the deactivation cluster of experiment 1 and its homolog area in 
the left hemisphere; these volumes of interest are depicted in Figure $2 \mathrm{~A}$.

Figure 3 (middle) shows, for one subject, brain regions that exhibit phasic negative BOLD response to ipsilateral stimulation and tonic positive BOLD response to contralateral stimulation. The conjunction of contrasts [(left-hand, tonic) baseline] and [baseline - (right-hand, phasic)] was applied to detect such regions in the right SI cortex and the conjunction of [(right-hand, tonic) - baseline] and [baseline - (left-hand, phasic)] in the left SI cortex. Areas fulfilling these criteria appear in both hemispheres in the SI cortex of the postcentral gyrus.

We detected similar patterns in 7 of 10 subjects in the right SI cortex and in 7 of 10 in the left; 5 of 10 subjects featured such patterns in both hemispheres. Table 2 lists details of the detected clusters for each subject.

Figure 3 (outermost panels) shows the mean \pm SEM BOLD responses for those subjects who have statistically significant clusters in the respective areas. Statistically significant positive BOLD changes to contralateral stimulation are seen in both hemispheres (peak amplitudes, $0.84 \pm 0.13 \%$ in the right SI and $1.21 \pm 0.16 \%$ in the left SI; both signals differ from 0 at $p<$ $0.001)$. Correspondingly, negative BOLD changes are seen to ipsilateral stimulation (peak amplitudes, $-0.49 \pm 0.15 \%$ in the right SI and $-0.52 \pm 0.15 \%$ in the left SI; both signals statistically significantly different from 0 at $p<0.01$ ).

\section{Areas exhibiting negative BOLD response to both ipsilateral and contralateral stimulation: experiment 2}

The contrast [(left-hand) - baseline $]+[$ (right-hand $)-$ baseline] demonstrated areas deactivated by both contralateral and ipsilateral stimulation. The yellow blobs in Figure 3 show such areas in the anterior bank of the central sulcus (MI cortex) in both hemispheres of one subject. We detected similar patterns in the right hemisphere in 6 of 10 subjects and in 6 of 10 in the left and 5 of 10 subjects featured such pattern in both hemispheres. Table 2 gives the sizes and coordinates of the detected clusters. The mean \pm SEM signal strengths as a function of time, shown in the top part of Figure 3, demonstrates negative BOLD responses to both left and right unilateral stimulation in both left and right MI cortices.

Tactile stimuli to the right-hand fingers elicited negative BOLD response with the peak amplitude of $-0.44 \pm 0.15 \%(p<$ 0.05 ) in the left (contralateral) MI cortex and with the peak amplitude of $-0.53 \pm 0.20 \%(p<0.05)$ in the right MI. Correspondingly, the stimuli to the left hand elicited negative BOLD responses in both the left $(-0.33 \pm 0.03 \% ; p<0.0001)$ and right $(-0.41 \pm 0.03 \% ; p<0.0001) \mathrm{MI}$ cortex.

\section{Discussion}

The present study demonstrates that unilateral tactile stimulation of fingers can be associated, in addition to the well known activation of the contralateral SI, with activation of area 2 and deactivation of area $3 \mathrm{~b}$ in the ipsilateral SI, as well as with deactivation of the MI cortex in both hemispheres. Both left- and right-sided stimulation of fingertips elicited tonic activation in the contralateral SI, whereas phasic deactivations were observed in the ipsilateral SI and in the MI cortices of both hemispheres.

Table 2. Details of detected clusters in experiment 2

\begin{tabular}{|c|c|c|c|c|}
\hline Subject & $x$ & $y$ & $z$ & Volume \\
\hline \multicolumn{5}{|l|}{ Right SI } \\
\hline s1 & $45 \pm 1.7$ & $-18 \pm 2.1$ & $48 \pm 3.7$ & 342 \\
\hline s2 & $34 \pm 2.6$ & $-23 \pm 1.7$ & $56 \pm 3.1$ & 190 \\
\hline s3 & $31 \pm 1.5$ & $-22 \pm 0.8$ & $67 \pm 2.1$ & 94 \\
\hline s5 & $36 \pm 3.2$ & $-22 \pm 3.5$ & $53 \pm 3.2$ & 528 \\
\hline s6 & $42 \pm 1.8$ & $-28 \pm 2$ & $51 \pm 1.6$ & 199 \\
\hline s7 & $44 \pm 1.7$ & $-24 \pm 2.7$ & $50 \pm 1.4$ & 197 \\
\hline s10 & $41 \pm 4.8$ & $-31 \pm 4.8$ & $57 \pm 3.8$ & 2137 \\
\hline Mean & $39 \pm 2$ & $-24 \pm 1.6$ & $55 \pm 2.4$ & $527 \pm 274$ \\
\hline \multicolumn{5}{|l|}{ Left SI } \\
\hline s1 & $-46 \pm 1.7$ & $-26 \pm 2.1$ & $52 \pm 3.6$ & 262 \\
\hline s3 & $-39 \pm 0.7$ & $-24 \pm 2$ & $54 \pm 1.3$ & 38 \\
\hline s4 & $-52 \pm 0.7$ & $-21 \pm 1.6$ & $55 \pm 1.4$ & 52 \\
\hline s5 & $-41 \pm 1.2$ & $-16 \pm 1.1$ & $60 \pm 1.6$ & 38 \\
\hline s7 & $-42 \pm 2.8$ & $-24 \pm 2.5$ & $54 \pm 2.3$ & 311 \\
\hline s8 & $-42 \pm 0$ & $-23 \pm 1.5$ & $49 \pm 0.4$ & 6 \\
\hline s10 & $-42 \pm 6.2$ & $-34 \pm 2.4$ & $52 \pm 3.1$ & 875 \\
\hline Mean & $-43 \pm 1.6$ & $-24 \pm 2.1$ & $54 \pm 1.3$ & $226 \pm 117$ \\
\hline \multicolumn{5}{|l|}{ Right MI } \\
\hline s1 & $24 \pm 2.2$ & $-29 \pm 1.8$ & $52 \pm 1.8$ & 210 \\
\hline s2 & $27 \pm 0.5$ & $-27 \pm 1.3$ & $52 \pm 0$ & 9 \\
\hline s5 & $17 \pm 0.9$ & $-25 \pm 1$ & $59 \pm 1.3$ & 47 \\
\hline s7 & $18 \pm 2.6$ & $-29 \pm 1.3$ & $60 \pm 1.7$ & 141 \\
\hline$s 9$ & $24 \pm 0.4$ & $-25 \pm 0$ & $58 \pm 1.6$ & 7 \\
\hline$s 10$ & $25 \pm 1.5$ & $-31 \pm 1.1$ & $66 \pm 1.5$ & 97 \\
\hline Mean & $23 \pm 1.6$ & $-28 \pm 1$ & $58 \pm 2.2$ & $85 \pm 33$ \\
\hline \multicolumn{5}{|l|}{ Left MI } \\
\hline s1 & $-32 \pm 2$ & $-28 \pm 0.9$ & $54 \pm 1.4$ & 94 \\
\hline s3 & $-16 \pm 1.3$ & $-26 \pm 1.4$ & $57 \pm 1$ & 71 \\
\hline s5 & $-29 \pm 3.3$ & $-22 \pm 1.3$ & $60 \pm 1.5$ & 228 \\
\hline s7 & $-22 \pm 3.4$ & $-26 \pm 2.9$ & $54 \pm 2.8$ & 806 \\
\hline s9 & $-27 \pm 2.2$ & $-25 \pm 0.6$ & $57 \pm 1.3$ & 64 \\
\hline s10 & $-39 \pm 1$ & $-21 \pm 1.5$ & $46 \pm 2.3$ & 64 \\
\hline Mean & $-28 \pm 3.3$ & $-25 \pm 1.1$ & $55 \pm 2$ & $221 \pm 120$ \\
\hline
\end{tabular}

Mean $\pm S D x, y$, and $z$ coordinates in Talairach space of voxels of the activated clusters in SI and MI for individual subjects listed in the left column. Volume gives the cluster size in cubic millimeters.

Occasional deactivation of the SI-MI region has been observed in numerous fMRI studies (Allison et al., 2000; Nirkko et al., 2001; Hamzei et al., 2002; Stefanovic et al., 2004; Newton et al., 2005). In the present study, we attempted to functionally and spatially differentiate the SI and MI cortices. Whereas experiment 1 allowed us to create an appropriate BOLD response model for the detection of phasic deactivation, deployment of this model in 
experiment 2 verified that during pure tactile stimulation phasic deactivation takes place, in addition to the ipsilateral SI (area 3b), also in the ipsilateral MI (area 4). In the analysis of experiment 2, we avoided any spatial smoothing to preserve spatial resolution and thereby to differentiate activations in the anterior and posterior bank of the central sulcus in the MI and SI cortices.

Ipsilateral responses to unilateral stimuli have been detected previously with intracortical electrophysiological recordings in Brodmann's area 1 (BA 1), BA 4, BA 2, and/or BA 7 (Allison et al., 1989) and with fMRI in BA 2 and/or BA 5 (Nihashi et al., 2005). In the present study, we detected activation in the ipsilateral postcentral sulcus, presumably in area 2. This finding agrees with previous monkey single-neuron recordings indicating bilateral hand representation in this region: Iwamura et al. (1994) found neurons with bilateral hand representation in the posterior bank of the postcentral gyrus in area 2 and along the ambiguous border between area 2 and areas 5/7.

\section{Transcallosal and corticocortical influences}

One plausible explanation for the present findings could be the following: immediately after activation of the contralateral area 3 by tactile input, areas 1 and 2 on the same side are activated via anteroposterior corticocortical projections. The ipsilateral SI areas could then obtain input through transcallosal connections, most likely via area 2, which was active in our study and has the densest transcallosal connections among all SI areas (Killackey et al., 1983). Area 2 also has reciprocal connections to area $3 \mathrm{~b}$ and dense connections to the motor cortex (Yumiya and Ghez, 1984). These connections could be responsible for the observed deactivations in the ipsilateral area $3 \mathrm{~b}$ and MI cortex. The well established, although sparse, transcallosal connections between the MI cortices (Jenny, 1979; Rouiller et al., 1994) could contribute as well. However, a transcallosal route originating from or affecting area $3 \mathrm{~b}$ directly is highly unlikely because hand representations in areas $3 \mathrm{~b}$ and 1 are practically free of transcallosal connections (Killackey et al., 1983).

Such interhemispheric SI-MI interaction could also account for the activation-deactivation patterns observed in previous studies (Allison et al., 2000; Nirkko et al., 2001; Hamzei et al., 2002; Stefanovic et al., 2004; Newton et al., 2005). Furthermore, the lack of area 2 neurons with bilateral receptive fields confined to the proximal forelimb (Iwamura et al., 1994) would explain the observations of Nirkko et al. (2001) of fMRI deactivation of the sensorimotor cortex for movements of distal but not proximal limbs.

In line with the importance of the transcallosal pathways for affecting the functional state of the ipsilateral SI cortex, Seyal et al. (1995) demonstrated lowered sensory thresholds, i.e., increased tactile sensitivity, when transcranial magnetic stimulation (TMS) was applied to the parietal cortex ipsilateral to the stimuli. The authors interpreted the finding as TMS-induced disruption of normally functioning interhemispheric inhibition. It is also possible to modulate interhemispheric inhibition by means of $1 \mathrm{~Hz}$ repetitive TMS (Pal et al., 2005). The TMS effects are in accordance with invasive animal studies: in monkeys, the receptive fields of area $3 \mathrm{~b}$ neurons expand after cooling of area $3 \mathrm{~b}$ in the opposite hemisphere (Clarey et al., 1996), and, in rats, interhemispheric inhibition is involved in modulating receptive fields in the SI cortex (Pluto et al., 2005).

\section{Mechanism of deactivation}

The BOLD signal has been demonstrated to correlate with the level of neuronal postsynaptic activity reflected in local field po- tentials (Logothetis et al., 2001; Mukamel et al., 2005; Niessing et al., 2005), suggesting that it primarily reflects neuronal input to the relevant cortical area and local processing rather than output activity (for review, see Logothetis and Wandell, 2004). Hence, an overall decrease of input and, accordingly, of neuronal activity in the studied brain area would appear as fMRI deactivation. A very recent study (appearing during the revision of this manuscript) actually verified by simultaneous fMRI and intracortical recordings the tight coupling between negative BOLD response and decreased neuronal activity in monkey primary visual cortex (Shmuel et al., 2006). Apparently, such a decrease of activity could result from presynaptic inhibition. The phasic time course of deactivation observed in our study (and also by Shmuel et al., 2006, their figures) is in line with this interpretation because IPSPs attenuate quickly (Deisz and Prince, 1989) and may even disappear at high stimulus repetition rates (Nacimiento et al., 1964).

Moreover, very recent intracortical recordings of multiunit activity and local field potentials in alert monkeys demonstrated inhibition in area $3 \mathrm{~b}$ in response to ipsilateral electrical mediannerve stimuli (Lipton et al., 2006). The fMRI recordings in the same study were unfortunately performed in anesthetized monkeys so that the results were not comparable with the electrophysiological recordings.

\section{Functional significance of deactivation}

In everyday life, people typically rely on information from onehand fingers when recognizing different textures. During such manual exploration, the texture affects all fingers of the same hand in a rather similar manner, whereas during tasks requiring bimanual manipulation, the two hands can receive very different input. In psychophysical studies, subjects perform better in comparing vibrotactile stimuli (Harris et al., 2001), and they recognize, identify, and discriminate patterns better (Craig, 1985; Craig and Qian, 1997) when the stimuli are presented to two fingers of opposite hands rather than to two fingers of the same hand. Accordingly, the observed simultaneous activation of the contralateral SI and deactivation of the ipsilateral SI could improve the left versus right differentiation of touch during cooperative bilateral hand actions. In line with these conjectures are findings that the tactile sensitivity in the right thumb decreases $(p<0.001)$ if $200 \mathrm{~ms}$ earlier a similar stimulus has been presented to the left thumb rather than to the left fifth finger; with a delay of $500 \mathrm{~ms}$, no such interference takes place (own additional analysis of the data of Braun et al., 2005).

In conclusion, we demonstrated phasic suppression in area $3 \mathrm{~b}$ of the ipsilateral SI cortex and in the MI cortices of both hemispheres in response to tactile stimuli. The negative BOLD response can be explained by decreased neuronal activity (cf. Shmuel et al., 2006) as a result of interhemispheric inhibition.

\section{References}

Allison JD, Meador KJ, Loring DW, Figueroa RE, Wright JC (2000) Functional MRI cerebral activation and deactivation during finger movement. Neurology 54:135-142.

Allison T, McCarthy G, Wood CC, Williamson PD, Spencer DD (1989) Human cortical potentials evoked by stimulation of the median nerve. II. Cytoarchitectonic areas generating long-latency activity. J Neurophysiol 62:711-722.

Boynton GM, Engel SA, Glover GH, Heeger DJ (1996) Linear systems analysis of functional magnetic resonance imaging in human V1. J Neurosci 16:4207-4221.

Braun C, Hess H, Burkhardt M, Wuhle A, Preissl H (2005) The right hand knows what the left hand is feeling. Exp Brain Res 162:366-373.

Clarey JC, Tweedale R, Calford MB (1996) Interhemispheric modulation of 
somatosensory receptive fields: evidence for plasticity in primary somatosensory cortex. Cereb Cortex 6:196-206.

Craig JC (1985) Attending to two fingers: two hands are better than one. Percept Psychophys 38:496-511.

Craig JC, Qian X (1997) Tactile pattern perception by two fingers: temporal interference and response competition. Percept Psychophys 59:252-265.

Deisz RA, Prince DA (1989) Frequency-dependent depression of inhibition in guinea-pig neocortex in vitro by GABAB receptor feed-back on GABA release. J Physiol (Lond) 412:513-541.

Genovese CR, Lazar NA, Nichols T (2002) Thresholding of statistical maps in functional neuroimaging using the false discovery rate. NeuroImage 15:870-878.

Grefkes C, Geyer S, Schormann T, Roland P, Zilles K (2001) Human somatosensory area 2: observer-independent cytoarchitectonic mapping, interindividual variability, and population map. NeuroImage 14:617-631.

Hamzei F, Dettmers C, Rzanny R, Liepert J, Buchel C, Weiller C (2002) Reduction of excitability ("inhibition") in the ipsilateral primary motor cortex is mirrored by fMRI signal decreases. NeuroImage 17:490-496.

Hari R, Imada T (1999) Ipsilateral movement-evoked fields reconsidered. NeuroImage 10:582-588.

Harris JA, Harris IM, Diamond ME (2001) The topography of tactile working memory. J Neurosci 21:8262-8269.

Hlushchuk Y, Hari R (2005) Phasic deactivation of human ipsilateral somatosensory cortex during tactile stimulation. Soc Neurosci Abstr 31:15.9.

Iwamura Y, Iriki A, Tanaka M (1994) Bilateral hand representation in the postcentral somatosensory cortex. Nature 369:554-556.

Jenny AB (1979) Commissural projections of the cortical hand motor area in monkeys. J Comp Neurol 188:137-145.

Kanno A, Nakasato N, Hatanaka K, Yoshimoto T (2003) Ipsilateral area 3b responses to median nerve somatosensory stimulation. NeuroImage 18:169-177.

Killackey HP, Gould III HJ, Cusick CG, Pons TP, Kaas JH (1983) The relation of corpus callosum connections to architectonic fields and body surface maps in sensorimotor cortex of new and old world monkeys. J Comp Neurol 219:384-419.

Korvenoja A, Wikström H, Huttunen J, Virtanen J, Laine P, Aronen HJ, Seppäläinen AM, Ilmoniemi RJ (1995) Activation of ipsilateral primary sensorimotor cortex by median nerve stimulation. NeuroReport 6:2589-2593.

Korvenoja A, Huttunen J, Salli E, Pohjonen H, Martinkauppi S, Palva JM, Lauronen L, Virtanen J, Ilmoniemi RJ, Aronen HJ (1999) Activation of multiple cortical areas in response to somatosensory stimulation: combined magnetoencephalographic and functional magnetic resonance imaging. Hum Brain Mapp 8:13-27.

Lipton ML, Fu KM, Branch CA, Schroeder CE (2006) Ipsilateral hand input to area $3 \mathrm{~b}$ revealed by converging hemodynamic and electrophysiological analyses in macaque monkeys. J Neurosci 26:180-185.

Logothetis NK, Wandell BA (2004) Interpreting the BOLD signal. Annu Rev Physiol 66:735-769.

Logothetis NK, Pauls J, Augath M, Trinath T, Oeltermann A (2001) Neuro- physiological investigation of the basis of the fMRI signal. Nature 412:150-157.

Mertens M, Lütkenhöner B (2000) Efficient neuromagnetic determination of landmarks in the somatosensory cortex. Clin Neurophysiol 111:1478-1487.

Mukamel R, Gelbard H, Arieli A, Hasson U, Fried I, Malach R (2005) Coupling between neuronal firing, field potentials, and FMRI in human auditory cortex. Science 309:951-954.

Nacimiento AC, Lux HD, Creutzfeldt OD (1964) Postsynaptic potentials of nerve cells of the motor cortex after electric stimulation of specific and nonspecific thalamic nuclei (in German). Pflügers Arch Gesamte Physiol Menschen Tiere 281:152-169.

Newton JM, Sunderland A, Gowland PA (2005) fMRI signal decreases in ipsilateral primary motor cortex during unilateral hand movements are related to duration and side of movement. NeuroImage 24:1080-1087.

Nichols T, Brett M, Andersson J, Wager T, Poline JB (2005) Valid conjunction inference with the minimum statistic. NeuroImage 25:653-660.

Niessing J, Ebisch B, Schmidt KE, Niessing M, Singer W, Galuske RA (2005) Hemodynamic signals correlate tightly with synchronized gamma oscillations. Science 309:948-951.

Nihashi T, Naganawa S, Sato C, Kawai H, Nakamura T, Fukatsu H, Ishigaki T, Aoki I (2005) Contralateral and ipsilateral responses in primary somatosensory cortex following electrical median nerve stimulation-an fMRI study. Clin Neurophysiol 116:842-848.

Nirkko AC, Ozdoba C, Redmond SM, Burki M, Schroth G, Hess CW, Wiesendanger M (2001) Different ipsilateral representations for distal and proximal movements in the sensorimotor cortex: activation and deactivation patterns. NeuroImage 13:825-835.

Pal PK, Hanajima R, Gunraj CA, Li JY, Wagle-Shukla A, Morgante F, Chen R (2005) Effect of low-frequency repetitive transcranial magnetic stimulation on interhemispheric inhibition. J Neurophysiol 94:1668-1675.

Pluto CP, Chiaia NL, Rhoades RW, Lane RD (2005) Reducing contralateral SI activity reveals hindlimb receptive fields in the SI forelimb-stump representation of neonatally amputated rats. J Neurophysiol 94:1727-1732.

Rouiller EM, Babalian A, Kazennikov O, Moret V, Yu XH, Wiesendanger M (1994) Transcallosal connections of the distal forelimb representations of the primary and supplementary motor cortical areas in macaque monkeys. Exp Brain Res 102:227-243.

Seyal M, Ro T, Rafal R (1995) Increased sensitivity to ipsilateral cutaneous stimuli following transcranial magnetic stimulation of the parietal lobe. Ann Neurol 38:264-267.

Shmuel A, Augath M, Oeltermann A, Logothetis NK (2006) Negative functional MRI response correlates with decreases in neuronal activity in monkey visual area V1. Nat Neurosci 9:569-577.

Stefanovic B, Warnking JM, Pike GB (2004) Hemodynamic and metabolic responses to neuronal inhibition. NeuroImage 22:771-778.

Talairach J, Tournoux P (1988) Co-planar stereotaxic atlas of the human brain: 3-dimensional proportional system: an approach to cerebral imaging. New York: Thieme Medical Publishers.

Yumiya H, Ghez C (1984) Specialized subregions in the cat motor cortex: anatomical demonstration of differential projections to rostral and caudal sectors. Exp Brain Res 53:259-276. 